\section{Lassitude 2000}

J'ai regagné mon domicile sous une pluie battante, ce lundi à $20.10 \mathrm{~h}$. Des cinq visites à domicile que je venais d'effectuer, trois m'avaient laissé un goût étrange, d'amertume et de tristesse.

La première patiente présentait une violente crise de migraines, sans ingestion possible de nourriture ni de médicaments. Lorsque sa fille présenta l'ordonnance de suppositoires d'Ergosanol ce fut pour apprendre qu'ils n'étaient plus dans le commerce, comme d'ailleurs les Cafergot $P B$ dont il restait heureusement une boîte au pharmacien. Suppression "économique»? Non, décision fédérale ...

Le second patient, très âgé et investigué dans un hôpital de la région pour une fibrose pulmonaire, sortait d'une réhabilitation dont il me fit une description plus proche de la convalescence que du traitement. Sur demande du médecin responsable de ce confortable institut, il quémandait une investigation pneumologique approfondie effectuée quelques semaines auparavant. Hypocondrie? Non, manque de transmission ou de lecture des documents ...

La troisième personne avait séjourné dans un hôpital spécialisé de haute technologie pour «difficulté à instiller des gouttes ophtalmiques à domicile». Sans suivi réel durant deux semaines dans une pension "court séjour", elle présentait trois jours après son retour un adème du poumon impressionnant. Décompensation cardiaque soudaine? Non, arrêt du Lasix sans raison documentée ...

Le monde médical n'est-il donc plus ce qu'il était et dois-je croire ces patients dont les réadaptations se résumeraient à vingt minutes de physio par jour, ces malades qui prétendent n'avoir vu le médecin qu'à deux reprises durant leur séjour? Ou les vertus imaginaires de ce programme PLAISIR, plaie chronophage de nos EMS, qui n'évoque à tout prendre que le 1984 de Georges Orwell, son Big Brother et sa Minute de la Haine ...?

Mes collègues préservent pourtant la même conscience professionnelle, les infirmières sont aussi attentives et soucieuses, les pharmaciens à l'écoute et les physiothérapeutes disponibles. Mais d'ukase en enquête, de restriction en prélèvement, de colloques en tables rondes, quelles que soient nos motivations, nous sommes tous las, et chaque jour un peu plus

- las de lutter pour la prise en charge des soins palliatifs quand d'un coup de plume populiste le gui, la poudre de mandragore et l'héroïne obtiennent leurs lettres de finance;

- las lorsque meurent les médicaments simples et efficaces sous la férule non-pratiquante des "pharmacologiquement corrects";

- las de chercher l'économicité du traitement lorsque les bien-portants ne visent plus que l'épargne;

- las de croire à notre vocation quand nos politiciens consacrent l'obligation de contracter et confient à l'incompétence le privilège du choix.

... et la lassitude, en rétrécissant le champ de notre intérêt, passera pour négligence!

Nos professions sont exigeantes de compétence et d'humanité; elles s'accommodent mal de l'arbitraire et la correction veut que les quelques manifestations syndicales s'effectuent sans préjudice pour les malades. Mais jusqu'à quand abusera-t-on de notre patience? Jusqu'à quand baisserons-nous la tête en maugréant? Notre pouvoir est considérable et nous feignons de l'ignorer, notre liberté est immense et nous feignons de nous soumettre mais n'est-ce pas ainsi que le rat ficela le lion, selon ce bon Monsieur de La Fontaine?

Il en faudra plus bien sûr pour que nous relevions la tête et, s'il est trop tard, nous nous retrouverons comme en France, en Belgique ou en Angleterre, là où un de mes patients place ce mot d'accueil dans la bouche de tous les médecins: "What do you want?", sous-entendant pilules, examen ou arrêt de travail ...

Notre lassitude est patente mais nous surprend, nous qui l'avions jugée indigne de la profession. Aussi ne rêvons plus aux années qui nous séparent de la retraite mais à celles qu'ils nous restent pour remettre le soin aux malades au centre des préoccupations de santé publique.

Dr Eric Rochat a.Conseiller aux Etats (VD) St-Légier 